\title{
Anticullo \\ La biblioteca electrónica universitaria: un modelo de gestión
}

\author{
Por Eugenio Tardón
}

Resumen: Se propone la creación de un nuevo modelo de gestión y funcional para la biblioteca electrónica universitaria que tiene en cuenta tanto la gestión de los contenidos como de los agentes del conocimiento (profesores, investigadores y estudiantes) en el ámbito académico. Según este modelo, definir y manejar escenarios de intercambio que faciliten la creación, la difusión y el uso del conocimiento es el nuevo papel de la biblioteca

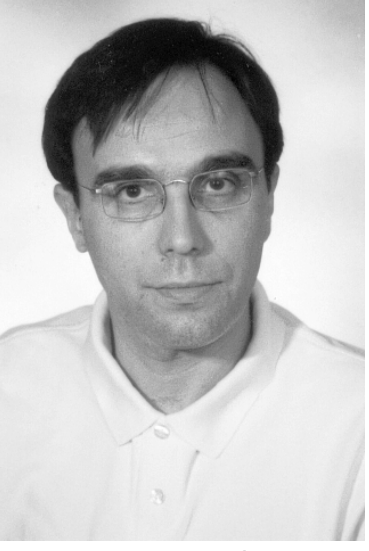

Eugenio Tardón electrónica en la era actual. Así se desprende del examen de su construcción histórica, sus herramientas y modelos para gestionar los procesos de transferencia de conocimientos que tienen lugar en su interior.

Palabras clave: Biblioteca electrónica, Historia, Herramientas de gestión, Gestión del conocimiento.

Title: Electronic academic libraries: a management model

Abstract: The development of a new management and functional model for the electronic academic library is presented, taking into account the management of both content and knowledge agents (i.e. scholars, researchers and students). This model proposes that the new role of the electronic library is to define and manage interchangeable scenarios for facilitating the creation, dissemination and use of knowledge. This is based on the history of the library, as well as on its tools and models for managing the processes of knowledge transfer that take place within it.

Keywords: Electronic library, history, Management tools, Knowledge management.

Tardón, Eugenio. "La biblioteca electrónica universitaria: un modelo de gestión". En: El profesional de la información, 2000, junio, v. 9, n. 6, pp. 18-24.

\section{Introducción}

Se examina en este trabajo la construcción histórica de la biblioteca electrónica universitaria y se presenta un nuevo modelo funcional y de gestión desde la perspectiva española, que tiene en cuenta las aportaciones de la sociología (en su análisis de la sociedad de la información) y de los teóricos del management en el ámbito de la gestión del conocimiento.

Se utilizará el concepto de biblioteca electrónica para referirse a una institución bibliotecaria que gestiona tanto materiales impresos como digitales y en la que el peso de estos últimos aumenta a ritmos muy elevados. Por los demás, es un término que ha calado profundamente en la sociedad y, desde el punto de vista del autor, tiene connotaciones más amplias que el de biblioteca híbrida. Mientras este calificativo se refiere sólo a los soportes documentales y a la relación información impresa/digital, el concepto de biblioteca electrónica denota, además de tipos de soporte, características estructurales (tanto de la información como de su gestión), que provocan transformaciones en los servicios y en la gestión de las bibliotecas.

El modelo pretende servir de herramienta para afrontar la gestión de las bibliotecas electrónicas universitarias en un momento, el actual, en el que sus funciones tradicionales como proveedoras de contenidos están también siendo asumidas por servicios de carácter comercial que compiten con ellas. Lo que este modelo resalta es que la biblioteca electrónica universitaria dispone de mecanismos y métodos que le permiten resistir esta competencia y seguir ofreciendo servicios de información y conocimiento en la era digital, eso sí, abandonando las posiciones tranquilas en las que se instalaba la biblioteca tradicional para dedicarse a la gestión no sólo de los contenidos, sino también de los agentes del conocimiento: profesores, investigadores y estudiantes.

\section{La nueva economía como economía del conocimiento y su impacto sobre las bibliotecas}

La XXX edición del Foro económico de Davos celebrado en enero de este año 2000 ha consagrado la conversión indiscutible de la élite del capitalismo mundial a la nueva economía. Este concepto resume una difusa idea de revolución económica a escala mundial caracterizada por un crecimiento no inflacionario de larga duración gracias a los incrementos de productividad que se derivan de la aplicación masiva de las tecnologías de la información y telecomunicaciones, de la hipercompetición global y de la soberanía del cliente. Hay tres aspectos de esta nueva economía que inciden directamente sobre las instituciones bibliotecarias:

- Casi todo lo que acontece en ella es configurado, influido y modelado por las tecnologías de la información, que han provocado una nueva revolución desde la década de los 70 puesto que, como ha demostrado Castells (1997), han introducido una disconti- 
nuidad en la base material de la economía, la sociedad y la cultura.

- Como resultado, las fronteras y las barreras entre productos y servicios, industrias, sectores, empresas, organizaciones y departamentos funcionales están siendo destruidas.

- Lo distintivo de esta economía es que la productividad y competitividad de los agentes económicos (los individuos, las organizaciones, las regiones y las naciones) dependen de su capacidad para generar, procesar y aplicar información basada en el conocimiento, lo que se consigue mediante el despliegue de tecnologías de la información y de las comunicaciones - tanto de infraestructuras como de máquinas y aparatos para procesar información (ordenadores, teléfonos, tarifa plana de acceso a la internet) — y unos recursos humanos bien adiestrados. Sin estos dos elementos, el potencial de las tecnologías de la información y comunicaciones se inhibe, pues no se produce el fenómeno de retroalimentación entre la acción innovadora y su uso.

\section{«La biblioteca electrónica uni- versitaria dispone de mecanis- mos y métodos que le permiten seguir ofreciendo servicios de información y conocimiento en la era digital, abandonando las posiciones tranquilas en las que se instalaba la biblioteca tradicional»}

Para responder a estos cambios del entorno y resistir su impacto, las instituciones bibliotecarias, sobre todo las del ámbito universitario (que han sido las más ágiles), han desarrollado dos estrategias:

1. Aumentar su adaptabilidad incrementando su tecnología y construyendo la biblioteca electrónica. El objetivo es hacerse más visibles y accesibles para no diluirse y desaparecer en ese nuevo espacio denominado tercer entorno (Echevarría, 1999).

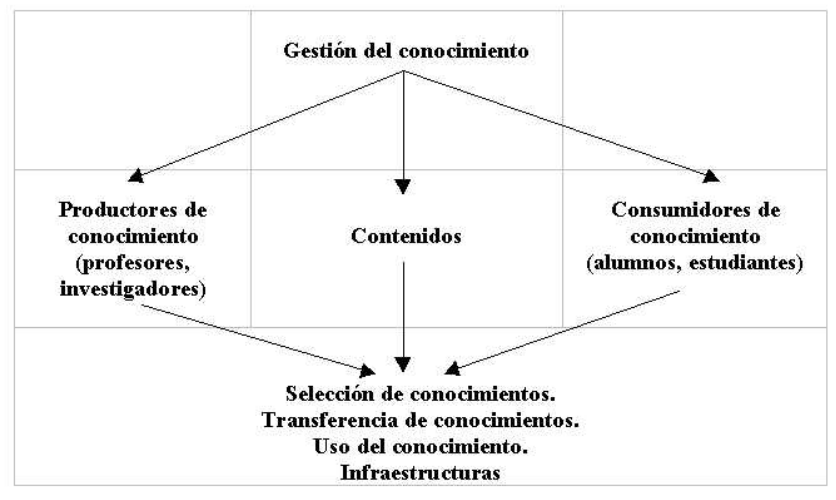

Figura 1. Modelo de biblioteca electrónica
2. Han redefinido sus funciones, sus competencias esenciales, sus core competences: de depósitos pasivos de conocimientos a servicios que transforman los datos almacenados en conocimiento para beneficio de sus usuarios pero que, al mismo tiempo, realizan la función inversa: transforman el conocimiento (depositado como información en los recursos) en datos almacenados para provecho de la posteridad y de la sociedad (Brophy, 1998). En resumidas cuentas, las bibliotecas se postulan como marcos de gestión de los procesos de transferencia del conocimiento.

Estas dos estrategias se funden en la creación de un nuevo modelo funcional y de gestión que es su principal herramienta para la acción en la era digital y que pretende conceptualizar el entorno para apropiarse de él, igual que el lenguaje hace con el mundo, y responder con eficacia a los retos que éste le plantea. Veamos cómo se ha desarrollado históricamente este modelo.

\section{La construcción de la biblioteca electrónica}

Hasta hace 20 años las bibliotecas custodiaban y organizaban sus colecciones (libros y revistas sobre todo), que eran el soporte del conocimiento científico y la cultura, de acuerdo con los principios de la biblioteconomía tradicional que permite acceder a los recursos de conocimiento hojeando directamente en las estanterías o consultando los catálogos de fichas.

La digitalización introduce cambios drásticos en la manera de acceder a los recursos. Lo primero que se digitaliza son los documentos secundarios, para construir un único catálogo que integre a los demás: el opac. Éste posibilita localizar información por cualquiera de los parámetros estructurados que describen los documentos primarios, sin necesidad de conocer la palabra por la que empieza el punto de acceso, puesto que se puede consultar por palabra clave.

Pero cuando el documento primario también se digitaliza, el antiguo corpus procedimental que constituía la tecnología bibliotecaria - los procedimientos de descripción bibliográfica, las herramientas de búsqueda y localización de información o los serviciosy en suma, todo el sistema, necesita reconfigurarse para funcionar en el nuevo entorno. Pero, ¿por qué ya no es válido lo anterior? Pues porque desaparece el espacio y todo tipo de límites y barreras espaciales:

-en primer lugar, las fronteras del soporte de almacenamiento: ya no hay libros, revistas, mapas, películas o fotos, todos son iguales: digitales. Lo único que cambia es el espacio de disco que ocupan según el tipo de información que contienen, y 


\begin{tabular}{|c|c|c|c|}
\hline & Profesores & Es tudiantes & Contenidos \\
\hline Profesores & $\begin{array}{l}\text { Paneles de } \\
\text { participación } \\
\text { temáticos. } \\
\text { Publicación de } \\
\text { documentación } \\
\text { multimedia en } \\
\text { servidores web } \\
\text { pers onales. } \\
\text { Actualizaciones de } \\
\text { documentos de trabajo, } \\
\text { de bibliografia. }\end{array}$ & $\begin{array}{l}\text { Expos iciones diferidas. } \\
\text { Expos iciones on-line. } \\
\text { Consulta online. } \\
\text { Publicación de notas de } \\
\text { interés. } \\
\text { Exámenes: archivos } \\
\text { anteriores, entrenamiento. }\end{array}$ & $\begin{array}{l}\text { 1. Todos los generados en cada } \\
\text { posible escenario. } \\
\text { 2. Contenidos actuales: } \\
\text { telememorias (webopac, } \\
\text { pas arelas temáticas, intranets, } \\
\text { servidores de ficheros, } \\
\text { carpetas públicas), préstamo, } \\
\text { servicios de actualización de } \\
\text { conocimiento, teledescarga de } \\
\text { documentos, etc. }\end{array}$ \\
\hline Es tudiantes & $\begin{array}{l}\text { Expos iciones diferidas. } \\
\text { Expos iciones on-line. } \\
\text { Consulta on-line. } \\
\text { Publicación de notas } \\
\text { de interés. } \\
\text { Exámenes: archivos } \\
\text { anteriores, } \\
\text { entrenamiento. }\end{array}$ & $\begin{array}{l}\text { Herramientas para crear } \\
\text { contenidos } \\
\text { individualmente. } \\
\text { Programas que } \\
\text { favorezcan la creación de } \\
\text { contenidos en grupos: } \\
\text { charlas, sistemas de } \\
\text { publicación, etc. } \\
\text { Depós itos para guardar } \\
\text { contenidos. } \\
\text { Herramientas para } \\
\text { aoceder a los depós itos } \\
\text { personales, etc. }\end{array}$ & $\begin{array}{l}\text { 1. Nuevos contenidos generados } \\
\text { en cada pos ible escenario. } \\
\text { 2. Contenidos actuales: } \\
\text { telememorias (webopac, } \\
\text { pas arelas temáticas, intranets, } \\
\text { servidores de ficheros, } \\
\text { carpetas públicas), préstamo, } \\
\text { servicios de actualización de } \\
\text { conocimiento, teledescarga de } \\
\text { documentos, etc. }\end{array}$ \\
\hline Contenidos & $\begin{array}{l}\text { 1. Nuevos contenidos } \\
\text { generados en cada } \\
\text { escenario pos ible. } \\
\text { 2. Contenidos actuales: } \\
\text { telememorias } \\
\text { (webopac, pas arelas } \\
\text { temáticas, intranets, } \\
\text { servidores de ficheros, } \\
\text { carpetas públicas), } \\
\text { préstamo, servicios de } \\
\text { actualización de } \\
\text { conocimiento, } \\
\text { teledescarga de } \\
\text { documentos, etc. }\end{array}$ & $\begin{array}{l}\text { 1.Nuevos contenidos } \\
\text { generados en cada } \\
\text { escenario pos ible. } \\
\text { 2. Contenidos actuales: } \\
\text { telememorias (webopac, } \\
\text { pas arelas temáticas, } \\
\text { intranets, servidores de } \\
\text { ficheros, carpetas } \\
\text { públicas), préstamo, } \\
\text { servicios de actualización } \\
\text { de conocimiento, } \\
\text { teledescarga de } \\
\text { documentos, etc. }\end{array}$ & \\
\hline
\end{tabular}

- también las barreras de depósito (de libros o de revistas). El único que permanece es el disco magnético u óptico.

El resultado es un incremento de la complejidad. Hay que tener en cuenta que en un sistema manual el entorno físico proporciona pistas para la acción. El control de los procesos se guía y orienta por el manejo de objetos físicos que se albergan en lugares concretos. Por ejemplo: papeles con anotaciones, facturas, libros, etc., que se guardan en el departamento de adquisiciones o el de materiales de referencia.

Pero cuando el entorno es electrónico las claves físicas ya no están presentes, se evaporan. No queda más remedio que moverse a través de un espacio vir- tual más complicado y difícil. Para poder transitar y trabajar en su interior, hay que empezar a rotularlo y a construir su mapa conceptual, lo que requiere reprogramar todos los conocimientos y utilizar nuevas herramientas lingüísticas, topográficas, arquitectónicas y conceptuales:

1. Hay que aprovechar las nuevas herramientas lingüísticas de recuperación de conocimientos derivadas de las posibilidades que ofrece la informática como soporte, lo que permite utilizar lenguajes de marcas (html, xml) para organizar los objetos digitales de información, visualizarlos de varias maneras y filtrarlos por tema o emisor. 
Si sus documentos son estratégicos para su compañia, también lo son para nosotros

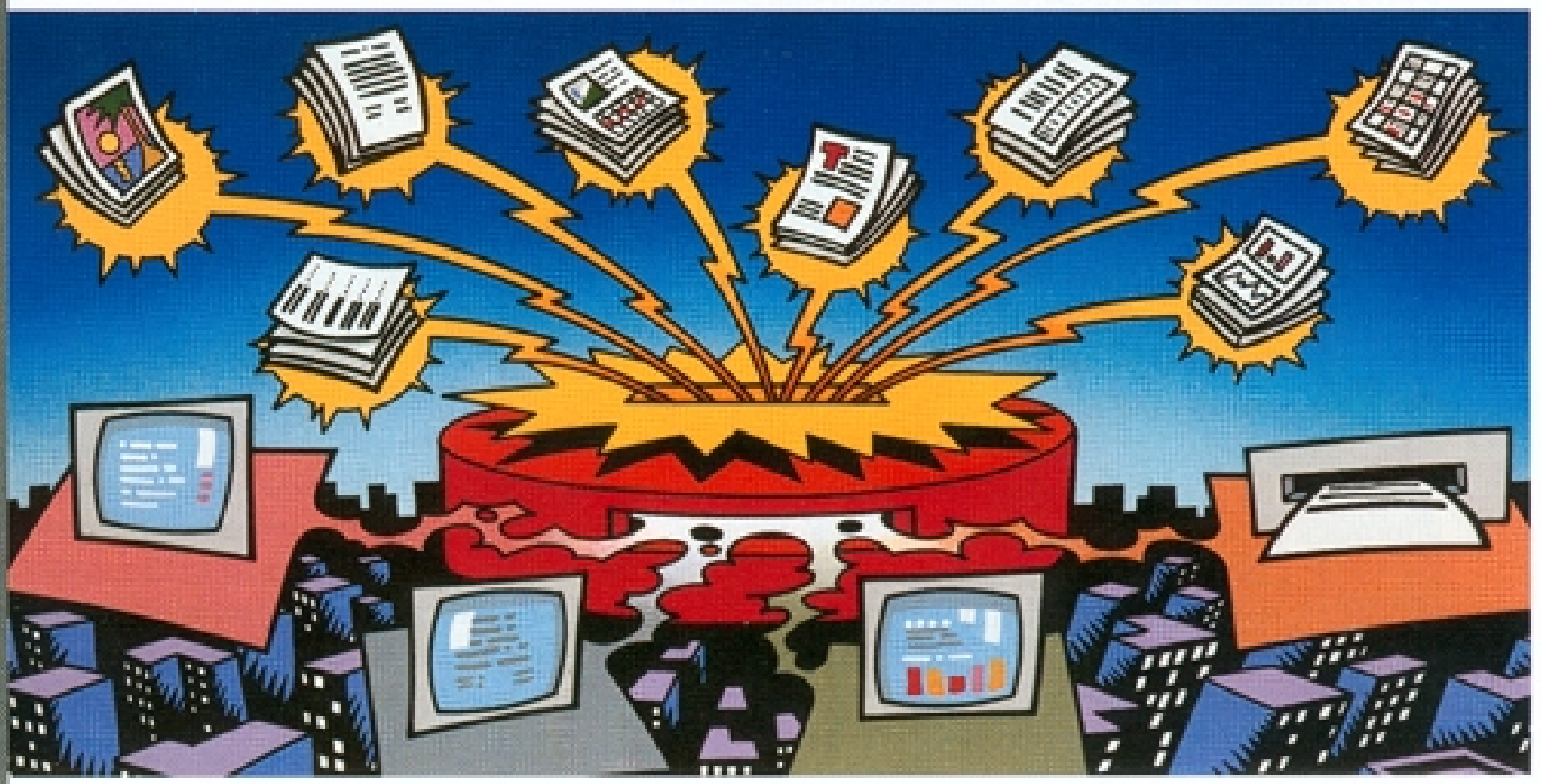

grosention

sempio

Exper

teision.

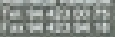

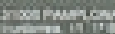

Amention

semescos

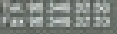

$\lim _{x \rightarrow 10}$

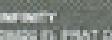

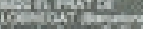

anisis

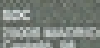

innsigy

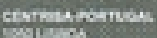

s.

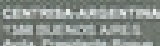

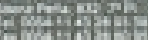

$\cos$

inconsting

costers

sines

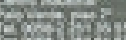

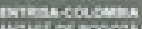

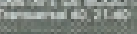

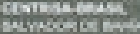

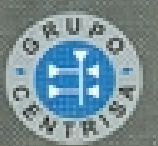

\section{4 -7RTLus}

\section{La Solución Documental}

BASISplus es el único sistema de gestión de documentos que permite gestionar de un modo integrado toda la información que circula por su organización.

Con BASISplus su trabajo será más fácil, su tiempo más rentable y su información más segura y accesible.

\section{TEEMILIBPLS}

\section{La Gestión de Bibliotecas}

TECHLIBplus es una solución completa totalmente diseñada bajo entorno Web para gestionar los recursos de información y actualizar el trabajo diario de las bibliotecas.

Aplicaciones basadas en las tecnologias BASISplus y TECHLIBplus:

Centros de Documentación

Sistemas de Gestión de Calidad

Gabinetes de Prensa

Difusión de Boletines Oriciales

Gestión de Expedientes

Normativa y Procedimientos

Producción Eclitorial

Gestion de Archivos
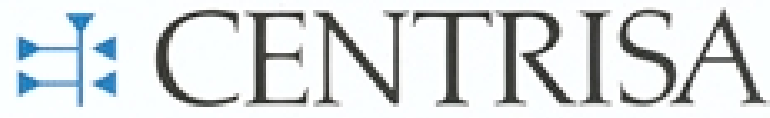

Tecnologias de la Informaciṕr 
2. Es necesario aprender a utilizar nuevas herramientas topográficas que ayuden a distribuir los recursos de conocimiento electrónico y a distinguir conceptualmente entre el propio recurso — que tiene asignada una dirección concreta en el espacio virtual: su URLy está compuesto por ficheros que pueden almacenar datos, documentos, catálogos, directorios, etc.; y su protocolo de acceso (http, ftp, z39.50).

\section{«Además de tramitar los conte- nidos, la biblioteca electrónica debe crear y gestionar merca- dos donde productores y con- sumidores intercambien cono- cimientos»}

3. Hay que construir una nueva arquitectura de la información con nuevas herramientas tecnológicas: los servidores web, las aplicaciones hipertextuales, los nuevos webopacs y los motores de búsqueda son las más conocidas. Esta estructura debe ayudar a vincular o conectar las necesidades de información con los recursos de conocimiento disponibles, tarea que requiere cartografiar temática y funcionalmente el espacio virtual en que se mueven los recursos de conocimiento y estructurarlos de forma que se pueda ofrecer a los usuarios un portal único de acceso a ellos.

4. Finalmente hay que utilizar herramientas conceptuales y elaborar un modelo de biblioteca electrónica que haga patente su carácter de institución orientada a la gestión y transferencia de conocimientos.

\section{El modelo de la gestión del conocimiento aplicado a la biblioteca electrónica}

Se ha afirmado anteriormente que las bibliotecas han redefinido sus core competences convirtiéndose en el marco para la gestión de los procesos de transferencia de conocimientos. ¿Qué es lo que legitima a la biblioteca para ocupar ese marco? Pues su propia historia, ya que la biblioteca es el sitio de los contenidos, el content site por excelencia. Para gestionarlos, la biblioteca electrónica tiene que construir un modelo funcional que exprese las operaciones que realiza sobre ellos para dar servicios a sus usuarios. Esas funciones son: seleccionar, transferir y usar conocimiento, proporcionar infraestructuras y acometer tareas de gestión y control. De forma detallada son las expuestas a continuación.

1. Selección de conocimiento. Actividades de identificación y organización de recursos de conocimiento, tanto externos como internos a la biblioteca, para crear almacenes de conocimiento o telememorias. Implica varias subactividades:

-Identificar recursos de conocimiento: lo que moviliza actividades de búsqueda, evaluación de su calidad y análisis de costes de cara a su adquisición. Una vez adquiridos, sea para poseerlos o para tener acceso a su empleo en virtud de una licencia, la biblioteca tiene que

- organizar los recursos de conocimiento, que conlleva actividades de descripción, análisis del contenido y estructuración de los mismos: temática, tipología, formatos, relaciones, etc. Es en esta labor donde la función de los bibliotecarios como creadores de conocimiento, incrementando su valor añadido, resulta más nítida pues su objeto es

- crear telememorias o almacenes de conocimiento, que satisfacen tres funciones: poner en contacto al usuario con la oferta de recursos, servir de instrumento para compartir conocimientos (en tanto telememorias colectivas de uso público), y facilitar la búsqueda, navegabilidad, uso y reutilización de recursos.

Estas telememorias constituyen el catálogo de recursos de conocimiento a los que la biblioteca da acceso, y adoptan distintos nombres en función del tipo de uso. Las principales son:

a. WebOpac: es la principal y, de hecho, el resto de telememorias no son sino subconjuntos que se integran en ella. Constituye el portal de acceso a todos los recursos de conocimiento de la biblioteca electrónica y es su instrumento de prestación de servicios.

b. Pasarelas temáticas: aquéllas que facilitan el acceso a recursos científicos en internet evaluados y seleccionados por su utilidad, relevancia y contenido temático.

c. Intranets: depósitos de conocimientos internos de la biblioteca que incluyen normas, políticas, noticias, cursos de formación y otros recursos.

d. Mapros: manuales de procedimientos que sirven para objetivar el conocimiento tácito mediante la formalización sistemática de las fases en que se descompone la elaboración de un servicio o producto. Su objeto es establecer una forma canónica de ejecución de estos servicios.

e. Bases de conocimiento: reúnen información sobre problemas encontrados en la prestación de servicios en las bibliotecas y la forma de resolverlos.

f. Servidores de ficheros y carpetas públicas.

2. Transferencia de conocimientos. Se refiere a las actividades de difusión de los recursos de conoci- 
miento. En la biblioteca electrónica es un proceso concatenado con el de selección de conocimientos, pues éstos se transfieren a través de los distintos servicios ofrecidos. Entre ellos se encuentran el webopac, los sistemas de actualización de conocimientos y el préstamo.

3. Uso de los recursos. Mediante esta función los usuarios explotan los contenidos a través de herramientas tecnológicas (procesadores de texto, hojas de cálculo, software de bases de datos, visualizadores estándar o especiales del tipo Acrobat). Ésta es una situación relativamente nueva, pues ahora su explotación está mediatizada por el empleo de tecnologías que actúan como prótesis intermediarias entre el contenido y el receptor. Un ejemplo es el siguiente: un usuario necesita consultar información de tipo estadístico sobre la UE y selecciona una base de datos estadística obteniendo los datos que precisa. Como no sólo quiere datos sino series y tiene que realizar análisis históricos y de tendencias, los exporta para su examen en una hoja de cálculo para, posteriormente, ejecutarla con el fin de llevar a cabo la tarea que le ha motivado al uso de los recursos de conocimiento de la biblioteca.

\section{«La biblioteca electrónica uni- versitaria resultante requiere un nuevo modelo funcional $y$ de gestión que aproveche las ca- racterísticas estructurales del espacio digital»}

4. Infraestructuras. La biblioteca electrónica proporciona las infraestructuras y servicios de apoyo que los usuarios necesitan para explotar el conocimiento. Por un lado, infraestructura de redes de ordenadores y medios de impresión, además de lugares de consulta y estudio y, por otro, servicios de asesoramiento personal: de uso de tecnologías y de utilización de recursos de conocimiento fundamentalmente.

5. Actividades de gestión. La función de gestión de cualquier organización debe asegurar que todos sus fondos se empleen de manera eficaz y, al mismo tiempo, seleccionar los indicadores de resultados que van a utilizarse de cara a evaluar la actividad de la biblioteca.

Pero este modelo estaría incompleto si no contemplara los tres elementos de la matriz de relaciones del conocimiento en el entorno académico: los contenidos principalmente, pero también los agentes del conocimiento, es decir, los productores (profesores, investigadores) y los consumidores (alumnos, estudiantes, así como otros profesores).

Pues bien, además de tramitar los contenidos, la biblioteca electrónica debe crear y gestionar mercados donde productores y consumidores intercambien conocimientos. Esos mercados son de naturaleza virtual, son espacios o escenarios que facilitan la creación de materiales y permiten el intercambio y la comunicación entre profesores, entre alumnos y entre ambos colectivos. Por otra parte, la gestión de este contexto comporta también actividades funcionales como las descritas para los contenidos y que se harán más explícitas a medida que se desarrollen dichos escenarios. He aquí algunas propuestas de mercados de servicios para los agentes del conocimiento:

\section{Para la relación profesor-profesor:}

- Servicio de paneles de participación o grupos de discusión temáticos para intercambiar información entre profesores o investigadores de áreas de trabajo próximas,

\section{Próximos números monográficos}

$\begin{aligned} & \begin{array}{r}\text { Septiembre 2000 } \\ \text { Diciembre 2000 }\end{array} \begin{array}{l}\text { Consultores en recursos de información } \\ \text { Información en ciencias de la salud }\end{array} \\ & \text { Marzo 2001 } \begin{array}{l}\text { Hosts y productores de bases de datos } \\ \text { Mayo 2001 }\end{array} \\ & \text { Información para la inteligencia competitiva }\end{aligned}$

Los interesados pueden remitir notas, artículos, propuestas, publicidad, comentarios, etc., sobre estos temas a:

epi@sarenet.es 
—servicio de publicación de documentación multimedia en servidores web personales, y

- servicio de actualizaciones de documentos de trabajo y bibliografía.

2. Para la relación profesor-alumno y viceversa los servicios serían:

-de exposiciones diferidas: lecciones y conferencias del profesorado accesibles vía red,

-de exposiciones online: lecciones o conferencias en directo, démicas,

- de consulta online para resolver cuestiones aca-

—de publicación de notas de interés relacionadas con los temas expuestos en clase: noticias de prensa, radio, etc., $\mathrm{y}$

—de exámenes o evaluaciones: archivos de exámenes, herramientas para crearlos, entrenamiento para aprobarlos (por ejemplo, las 1.500 preguntas o problemas anteriores de una materia).

\section{Para la relación alumno-alumno:}

-herramientas para crear contenidos individualmente (resúmenes, trabajos, tratamiento de datos, etc.),

-herramientas que favorezcan la creación de contenidos en grupos de trabajo o el intercambio de materiales sobre los contenidos, por ejemplo, charlas y sistemas de publicación, y

—depósitos para guardar contenidos.

Algunos podrían considerar que estas propuestas invaden el espacio de otros servicios educativos. No es ese el interés de este modelo de gestión. En realidad, lo que se quiere subrayar es la polifuncionalidad de las infraestructuras y servicios que puede ofertar la biblioteca electrónica universitaria aprovechando las oportunidades brindadas por las características estructurales del entorno digital: distalidad, reticularidad, multicronicidad, memoria externa e interdependencia, entre las más sobresalientes. (Echevarría, 1999)

\section{Conclusión}

La creciente densidad electrónica de la información utilizada en los campus universitarios ha convertido sus bibliotecas en bibliotecas electrónicas caracterizadas por un empleo intensivo de tecnologías de la información y las comunicaciones. En el espacio temporal de este tránsito, los centros han pasado por un proceso de realfabetización informacional para poder afrontar los cambios.
La biblioteca electrónica universitaria resultante requiere un nuevo modelo funcional y de gestión que aproveche las características estructurales del espacio digital y se concrete en nuevos servicios que tengan en cuenta la gestión de los contenidos y de los agentes del conocimiento en el ámbito académico (profesores, investigadores y estudiantes).

Las propuestas de servicios y de escenarios de intercambio apuntadas, a las que pueden añadirse muchas más en cuanto se reflexione conjuntamente con los agentes del conocimiento, constituirían, junto con los servicios desarrollados sobre los contenidos, la oferta global de servicios de la biblioteca electrónica. Una propuesta que pretende vincularla y fidelizarla a la comunidad académica sobre la base de los servicios que se ofrecen y que añaden valor a los tres elementos de la matriz de relaciones del conocimiento: productores, consumidores y contenidos.

En resumidas cuentas, no se trata de hacer lo mismo que antes pero en internet. Se trata, por encima de todo, de añadir valor al conocimiento y de gestionarlo, puesto que gestionar conocimiento en la biblioteca electrónica consiste en definir y manejar escenarios de intercambio para potenciar su creación, difusión y uso.

\section{Bibliografía}

Brophy, Peter. "Libraries without walls: from vision to reality". En: Brophy, Peter; Fisher; Clarke, Zoë. Libraries without walls 2: the delivery of library services to distant users. London: Library Association $\mathrm{Pu}-$ blishing, 1998.

Brophy, Peter; Wynne, Peter M. Management information systems and performance measurement for the electronic library: eLib supporting study (Miel2). Final report. 1997. Consultado en: 14-10-1999.

http://www.ukoln.ac.uk/dlis/models/studies/mis/mis.rtf

Castells, Manuel. La era de la información: economía, sociedad, cultura. Vol. 1: la sociedad red. Madrid: Alianza, 1997.

Davenport, Thomas H.; Prusak, Laurence. Information ecology: mastering the information and knowledge environment. New York: Oxford University Press, 1997.

Davenport, Thomas H.; Prusak, Laurence. Working knowledge: how organizations manage what they know. Boston: Harvard Business School, 1998.

Dutton, William H. (ed.). Society on the line: information politics in the digital age. New York: Oxford University, 1999.

Dempsey, Lorcan. "The network and the library: working in a new shared space: infrastructure and institutions". En: The electronic library, 1999, v. 17, n. 4, pp. 207-210.

Echevarría, Javier: Los señores del aire: telépolis y el tercer entorno. Barcelona: Destino, 1999.

Eugenio Tardón. Biblioteca Europea. Universidad Complutense de Madrid.

tardon@buc.ucm.es 